\title{
Hepatotoxicity in hyperthyroid patient after consecutive methimazole and propylthiouracil therapies
}

\author{
Fernando Gomez-Peralta', Pablo Velasco-Martínez², Cristina Abreu1', María Cepeda² \\ and Marta Fernández-Puente ${ }^{2}$
}

${ }^{1}$ Endocrinology and Nutrition Unit and 2Department of Internal Medicine, Hospital General de Segovia, Segovia, Spain
Correspondence should be addressed to F Gomez-Peralta Email

fgomezperalta@gmail.com

\section{Summary}

Methimazole (MMI) and propylthiouracil (PTU) are widely used antithyroid drugs (ATD) that have been approved for the treatment of hyperthyroidism. Hepatotoxicity may be induced by these drugs, though they exert dissimilar incidence rates of hepatotoxicity and, possibly, with different underlying pathogenic mechanisms. We report the case of a 55-yearold woman with no relevant medical history diagnosed with hyperthyroidism due to Graves' disease, who developed two episodes of acute hepatitis concurrent with the consecutive administration of two different ATDs, first MMI and then PTU. Given the impossibility of administering ATDs, it was decided to perform a total thyroidectomy because the patient was found to be euthyroid at that point. Pathological anatomy showed diffuse hyperplasia and a papillary thyroid microcarcinoma of $2 \mathrm{~mm}$ in diameter. Subsequent clinical check-ups were normal. This case suggests the importance of regular monitoring of liver function for hyperthyroid patients. Due to the potential severity of this side effect, it is recommended to determine baseline liver function prior to initiation of treatment.

\section{Learning points:}

- We present a rare case of two acute hepatitis episodes concurrent with two different consecutive ATD therapies.

- Our results highlight the relevance of a liver function monitoring during the treatment with MMI or PTU.

- A baseline assessment of the liver function before starting an ATD treatment should be recommendable.

\section{Background}

Liver dysfunction is a complication that can be observed in hyperthyroidism as a result of the use of antithyroid drugs (ATD). Although the overall incidence of hepatotoxicity induced by any antithyroid medication is $<0.5 \%$, when we focus on the most widely used drugs, the rate of liver dysfunction, including mild elevation of transaminase, rise by $15 \%(1,2)$. These common ATDs are methimazole (MMI) and propylthiouracil (PTU). Despite their relatively safe profiles, both can induce hepatotoxicity. Here, we report the case of a patient with Graves' disease who experienced two episodes of acute hepatitis concurrent with two different consecutive ATD therapies, an uncommon event that complicated patient management.

\section{Case presentation}

A 55-year-old woman with no relevant medical history was diagnosed as having hyperthyroidism due to Graves' disease based on classic clinical features and compatible laboratory test abnormalities (see Investigation section). 
Table 1 Laboratory results.

\begin{tabular}{|c|c|c|c|c|c|c|c|c|c|}
\hline Antithyroid treatment & $\begin{array}{c}\text { MMI start* } \\
\text { (Day 0) }\end{array}$ & $\begin{array}{l}\text { MMI stop } \\
\text { (Day 14) }\end{array}$ & $\begin{array}{c}\text { No } \\
\text { (Day 21) }\end{array}$ & $\begin{array}{c}\text { PTU start }^{+} \\
\text {(Day 28) }\end{array}$ & $\begin{array}{l}\text { PTU stop } \\
\text { (Day 56) }\end{array}$ & $\begin{array}{c}\text { No } \\
\text { (Day 64) }\end{array}$ & $\begin{array}{c}\mathbf{N o}^{\mathbf{}} \\
\text { (Day 74) }\end{array}$ & $\begin{array}{c}\text { No } \Phi \\
\text { (Day 104) }\end{array}$ & Reference range \\
\hline $\mathrm{TSH}, \mu \mathrm{UI} / \mathrm{mL}$ & 0.003 & 0.004 & 0.002 & 0.002 & 0.101 & 0.832 & 0.033 & 0.032 & $0.55-4.78$ \\
\hline Free T4, pmol/L & 109.13 & 53.87 & 61.37 & 93.62 & 8.49 & 9.21 & 12.27 & 21.32 & $10.07-22.70$ \\
\hline Free T3, pmol/L & 30.72 & 15.57 & & & 3.79 & 5.44 & & 5.49 & $3.50-6.50$ \\
\hline Bilirubin, mg/dL & & 3.80 & 5.0 & 2.5 & 2.0 & 1.6 & 1.2 & 1.2 & $0.30-1.10$ \\
\hline $\mathrm{ALT}, \mathrm{U} / \mathrm{L}$ & & 200 & 203 & 39 & 671 & 521 & 52 & 28 & $10-32$ \\
\hline AST, U/L & & 334 & 352 & 81 & 928 & 1139 & 147 & 31 & $10-55$ \\
\hline GGT, U/L & & 207 & 259 & 135 & 103 & 298 & 159 & 37 & $9-36$ \\
\hline Alkaline phosphatase, U/L & & & 362 & 211 & 226 & 441 & 264 & 151 & $40-150$ \\
\hline Amylase, U/L & & 113 & & & & & & & $28-100$ \\
\hline
\end{tabular}

* $10 \mathrm{mg}$ tid; ${ }^{\dagger} 100 \mathrm{mg}$ tid; ${ }^{*}$ Before total thyroidectomy; $\Phi$ After total thyroidectomy

ALT, alanine aminotransferase; AST, aspartate aminotrasnferase; GGT, gamma-glutamyl transpeptidase.

\section{Investigation}

The patient's follow-up was assessed through laboratory tests performed during the study period. A summary of laboratory results and reference ranges is provided in Table 1, but most relevant results are described below.

The patient presented classic clinical features and abnormalities in the laboratory test (reference range): thyroid-stimulating hormone (TSH): 0.003 (0.55-4.78) $\mu \mathrm{UI} / \mathrm{mL}$, free thyroxine (FT4): 109.1 (10.07-22.70) $\mathrm{pmol} / \mathrm{L}$, free triiodothyronine (FT3): 30.7 (3.50-6.50) $\mathrm{pmol} / \mathrm{L}$, anti-TSH receptor antibodies: $11.18 \quad(<1.75)$ $\mathrm{UI} / \mathrm{mL}$ and undetectable anti-thyroglobulin and antithyroperoxidase antibodies. Thus, she was diagnosed as having hyperthyroidism due to Grave's disease and was prescribed with MMI for treatment it.

After 13 days with MMI, she came to the emergency department of our hospital complaining of asthenia, anorexia, abdominal pain, nausea and vomiting of 72-h duration. She had a fever of $38.3^{\circ} \mathrm{C}\left(100.9^{\circ} \mathrm{F}\right)$, manifest jaundice and pain upon palpation of the right hypochondrium without Murphy's sign or peritonism. An abdominal ultrasound was normal. The liver function test, which showed hypertransaminasemia with a mixed pattern (Fig. 1) and serologic tests of viral hepatitis (hepatitis B surface antigen (HBsAg), hepatitis B core total antibodies (anti-HBc), hepatitis C virus (HCV) antigen and hepatitis A total IgM antibody (HAVAB)), which were negative. Therefore, hepatotoxicity by MMI was suspected, and this treatment was discontinued. The liver function test results after MMI withdrawal returned to normal and treatment with PTU was decided to start.

One month later, the patient came for a medical check-up, and she was found completely asymptomatic and a test confirmed a controlled hyperthyroidism. However, liver function test showed increased transaminases levels with severe cytolysis (Fig. 1), test of autoantibodies associated to autoimmune hepatitis (antinuclear antibodies (ANA), smooth muscle antibodies (SMA), liver kidney microsomal type 1 (LKM-1) antibodies, antineutrophil cytoplasmic antibodies (ANCA)) and serological test of viral hepatitis (see before) were negative. Discontinuation of PTU administration was decided due to the diagnostic. After PTU withdrawal, liver function and thyroid function tests showed an improvement reaching values inside the normal range.

\section{Treatment}

She started treatment with both methimazole (MMI) (10 mg tid) and propranolol (10 mg tid). Thirteen days after

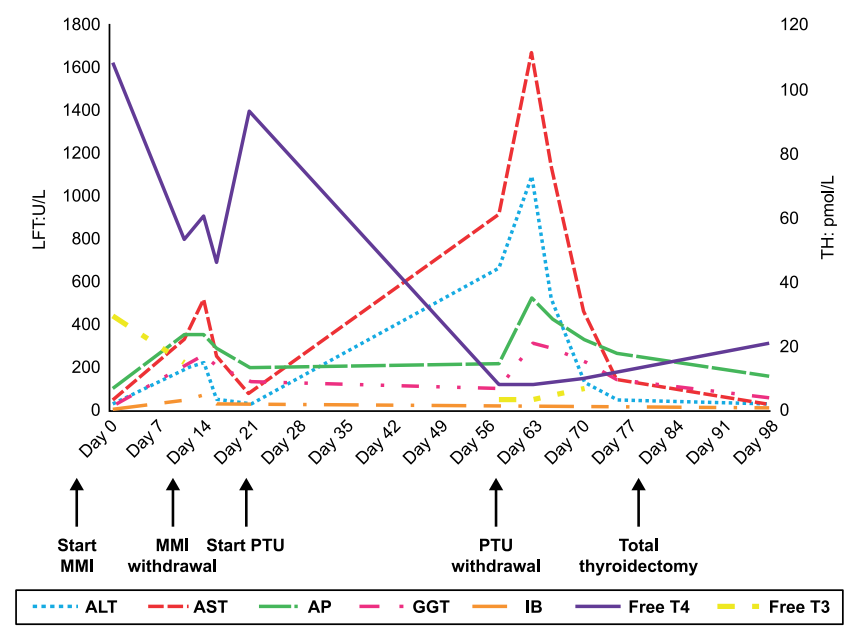

Figure 1

Monitoring of patient's molecular markers in liver and thyroid during the study. Alanine aminotransferase (ALT); Aspartate aminotransferase (AST); Alkaline phosphatase (AP); Gamma-glutamyl transferase (GGT); Indirect (unconjugated) bilirubin (IB); Free thyroxine (Free T4); Free triiodothyronine (Free T3); Liver function test (LFT); Thyroid hormones (TH). 
taking these drugs, a drug-induced hepatotoxicity by MMI was suspected. Therefore, MMI was discontinued. During the following 10 days, liver clinical signs and function test results returned to normal, so it was decided to start treatment with propylthiouracil (PTU) (100 mg tid). One month later, the patient came for a medical check-up, and she was found completely asymptomatic and a test confirmed a controlled hyperthyroidism. However, liver function test presented serious abnormalities with negative results of autoimmune and viral hepatitis tests (see Investigation section). This diagnostic led to the decision to discontinue PTU administration. After 8 days without treatment, the patient's liver function improved and her thyroid hormone levels remained in the normal range.

\section{Outcome and follow-up}

Finally, seven days after the last laboratory tests, it was decided to perform total thyroidectomy, carried out without complications. Pathological anatomy showed diffuse hyperplasia and a papillary thyroid microcarcinoma of $2 \mathrm{~mm}$ in diameter. Subsequent clinical check-ups were normal.

\section{Discussion}

The singularity of our case was the succession of two episodes of acute hepatitis concurrent with the consecutive administration of MMI and PTU therapies. As far as we know through literature review, there is no similar case published to date. The coincidence in time between the start of the drugs and the development of hepatitis, along with the improvement after drug discontinuation, provided us with a diagnostic clue. The initial differential diagnosis mainly included autoimmune hepatitis, hepatic dysfunction secondary to hyperthyroidism and viral hepatitis. The normality of the additional tests supported the suspicion of drug hepatotoxicity. Paradoxically, the symptoms were more intense with MMI despite less altered liver function.

Given the impossibility of administering an ATD, it was decided to perform a total thyroidectomy because the patient was found to be euthyroid at that point. The use of radioactive iodine would have been another reasonable alternative, but such therapy was considered inadvisable because of the need to achieve rapid control of hyperthyroidism without using an ATD.

ATDs are chemically thionamide derivatives (3). The most commonly used are MMI, carbimazole (which is not active by itself, but is virtually completely converted to MMI) and PTU. Hepatotoxicity may be induced by these two major thionamides, though MMI/carbimazole and PTU exert dissimilar hepatotoxicity levels, as was confirmed during the monitoring of this case (Fig. 1). In this line, Nakamura and coworkers (2) showed that PTU induced four times higher mild liver damages than MMI, at the same daily dose used in our study. MMI/ carbimazole is associated in a dose-dependent manner with an increased risk for hepatitis, while a role of the immune system might be involved in the pathogenesis of liver damage associated with PTU therapy, which seems to exhibit a more severe hepatotoxic profile in paediatrics $(1,4)$.

The underlying pathogenic mechanisms of ATDinduced hepatotoxicity are not yet fully understood. The significance of reactive metabolites in the pathogenesis of drug-induced hepatotoxicity has been described (3). The cytochrome P450 system is the major route of metabolism of $\mathrm{MMI} /$ carbimazole. MMI metabolism through CYP450 enzymes yields N-methylthiourea and glyoxal as two major metabolites, which have been suggested to be the basis for liver damage $(3,5)$. Biopsy specimens from patients with MMI-induced hepatic injury showed oedema and inflammation of the portal tract with intracanalicular cholestasis, typical of a cholestatic process, producing the typical cholestatic profile $(3,5)$. The onset of hepatotoxicity generally appears an average of 36 days (6) after the initiation of MMI/carbimazole.

Since glucuronidation is a one of the metabolic pathways for PTU, a proposed mechanism for liver damage induced by PTU could be attributed to its impaired detoxification process, due to lower activity of uridine diphosphoglucuronosyl transferase enzymes (UGTs) $(3,5)$. In this case, biopsy specimens from human subjects revealed that PTU caused parenchymal necrosis with haemorrhage, collapse of lobular architecture and preportal mixed inflammatory infiltrate, explaining a predominance of cytolysis $(3,5)$. In addition, immunemediated reactions are suggested to play a role in ATDinduced hepatotoxicity (5). Autoantibodies have been detected in some patients at the time of the acute episode, making it difficult to differentiate between a chronic autoimmune hepatitis and a PTU-mediated immune hepatitis (1). The onset of symptoms appears an average of 120 days (6) after the initiation of PTU.

Factors contributing to increased susceptibility to liver damage caused by ATDs are age (advanced age in the case of MMI/carbimazole and paediatric age in the case of PTU), female gender, the presence of pre-existing liver 
diseases and concomitant use of other hepatotoxic drugs (5). Due to the potential severity of this side effect, it is recommended to determine baseline liver function prior to initiation of treatment and to inform patients of the possible alarm symptoms (7). It remains to be evaluated if monitoring transaminases routinely is cost-effective to prevent cases of severe liver toxicity, as mild and reversible alterations of liver function tests are common in these patients (secondary to ATD treatment or due to hyperthyroidism itself (8)) and do not predict severe drug hepatotoxicity. Therefore, some authors argue that this practice could lead to unjustified discontinuation of ATDs (1). MMI/carbimazole and PTU are generally avoided in patients with baseline levels of transaminases higher than 3-5 times the upper limit of normal values not attributable to thyrotoxicosis (7).

The case reported here highlights the importance of regular monitoring of liver function for hyperthyroid patients who experienced liver dysfunction under MMI and later started PTU therapy, especially during the first month after initiating the second therapy. Such close monitoring will enable early recognition and treatment when hepatic abnormalities occur and prevent the progression of liver lesions.

We may conclude that although the prevalence of hepatotoxicity caused by MMI or PTU is extremely low, it must be known and suspected on the basis of compatible clinical and laboratory findings. Liver function monitoring during MMI or PTU therapy is warranted in patients with known risk factors.

\section{Declaration of interest}

The authors declare that there is no conflict of interest that could be perceived as prejudicing the impartiality of the research reported.

\section{Funding}

This research did not receive any specific grant from any public funding agency, commercial or not-for-profit sector.

\section{Patient consent}

Written consent has been obtained from the patient for publication of this article.

\section{Author contribution statement}

F G P and P V M were involved in data collection, patient care and followup. M C and M F P were involved in data collection and hospital care. $F$ G $P, M C$ and $M F P$ were involved in patient assessment, diagnosis and treatment. F G P, P V M and C A were major contributors in writing the manuscript. All authors read and approved the final manuscript. Medical writing support was provided by Antonio Torres-Ruiz at Dynamic Science S.L.

\section{References}

1 Akmal A \& Kung J. Propylthiouracil, and methimazole, and carbimazole-related hepatotoxicity. Expert Opinion on Drug Safety 201413 1397-1406. (https://doi.org/10.1517/14740338.2014.953 796)

2 Nakamura H, Noh JY, Itoh K, Fukata S, Miyauchi A \& Hamada N. Comparison of methimazole and propylthiouracil in patients with hyperthyroidism caused by Graves' disease. Journal of Clinical Endocrinology and Metabolism 200792 2157-2162. (https://doi. org/10.1210/jc.2006-2135)

3 Heidari R, Niknahad H, Jamshidzadeh A, Eghbal MA \& Abdoli N. An overview on the proposed mechanisms of antithyroid drugs-induced liver injury. Advanced Pharmaceutical Bulletin 20155 1-11. (https://doi.org/10.5681/apb.2015.001)

4 Wang MT, Lee WJ, Huang TY, Chu CL \& Hsieh CH. Antithyroid drugrelated hepatotoxicity in hyperthyroidism patients: a populationbased cohort study. British Journal of Clinical Pharmacology 201478 619-629. (https://doi.org/10.1111/bcp.12336)

5 Heidari R, Niknahad H, Jamshidzadeh A \& Abdoli N. Factors affecting drug-induced liver injury: antithyroid drugs as instances. Clinical and Molecular Hepatology 201420 237-248. (https://doi. org/10.3350/cmh.2014.20.3.237)

6 Burch HB \& Cooper DS. Management of Graves disease: a review. JAMA 2015314 2544-2554. (https://doi.org/10.1001/ jama.2015.16535

7 Bahn Chair RS, Burch HB, Cooper DS, Garber JR, Greenlee MC, Klein I, Laurberg P, McDougall IR, Montori VM, Rivkees SA, et al. Hyperthyroidism and other causes of thyrotoxicosis: management guidelines of the American Thyroid Association and American Association of Clinical Endocrinologists. Thyroid 201121 593-646. (https://doi.org/10.1089/thy.2010.0417)

8 Cooper DS. Antithyroid drugs. New England Journal of Medicine 2005 352 905-917. (https://doi.org/10.1056/NEJMra042972)

Received in final form 14 December 2017 Accepted 20 December 2017 